\title{
Direct observation of the impact of water droplets on oil replenishment in EHD lubricated contacts
}

\author{
Xiang LIU ${ }^{1}$, Jintao WANG ${ }^{1}$, Lu HUANG ${ }^{1, *}$, Jingyue ZHANG ${ }^{1}$, Changhong XU ${ }^{1}$, Lin TONG ${ }^{1}$, Dan GUO ${ }^{2, *}$ \\ ${ }^{1}$ National Institute of Metrology, Beijing 100029, China \\ ${ }^{2}$ State Key Laboratory of Tribology, Tsinghua University, Beijing 100084, China \\ Received: 15 May 2020 / Revised: 26 August 2020 / Accepted: 20 October 2020 \\ (C) The author(s) 2020 .
}

\begin{abstract}
Water is one of the most significant causes of lubrication failure. There is little research on the direct observation of the impact of water on lubrication properties. In this study, the influence of water on oil replenishment under different elastohydrodynamic (EHD) lubricating conditions is evaluated using optical interferometry and infrared microscopy, and a dimensionless criterion when water influences the film thickness is proposed. Evidence shows that the scour displacing effect and emulsification of water/oil are the main reasons for the reduction in film thickness. Once a water droplet enters an oil reservoir around the critical contact zone, it hardly moves away. This aggravates starvation, reduces the center film thickness of the contact, and leads to lubrication failure of the mechanical components.
\end{abstract}

Keywords: free water droplet; point contact; starved condition; film thickness

\section{Introduction}

Research shows that particles, moisture, and other pollutants can cause severe damage to rolling bearings, and $25 \%$ of the early failure of bearings is caused by the pollution of the lubricating oil systems [1-3]. To achieve long-term stable operation for offshore fans, dental drilling machines, and other machines working in humid environments, we must pay special attention to the effect of water on the lubrication system $[4,5]$. The sources of water in oil products include water entering because of poor sealing of the mechanical system, and the condensate produced because of the cold and hot alternate cycles during the start and stop of the mechanical system.

Three forms of water exist in lubricating and hydraulic oil: dissolved water, free water, and emulsified water. The solubility of water in lubricating oil depends on the type of base oil and additives. For most lubricating oils, the solubility of water is usually 400-600 ppm. Dissolved water means that the water content is less than the saturation of the lubricating oil, and the oil is in a transparent state; therefore, it cannot be judged visually that the lubricating oil is polluted by water. When the water content in the lubricating oil exceeds saturation, or additional water droplets enter the oil, the mixture forms micro-sized water/oil emulsions or macro-sized free water droplets. In some applications, water/oil emulsions are used as special lubricants. The flow and film formation mechanism of water/oil emulsions in an elastohydrodynamic (EHD) lubricating contact has been studied extensively by Wan et al. [6, 7]. However, few studies on the effect of visible free water droplets on lubrication have been reported. Generally, the amount of dissolved water is relatively small, which has a low impact on the performance of lubricating oil. Water-in-oil emulsions are usually synthesized for special application purposes. The only problem that needs to be studied further is the existence of undesired water droplets that occur in contact. The existence of this type of water is very dangerous not only for the lubricant but

* Corresponding authors: Lu HUANG, E-mail: huang1@nim.ac.cn; Dan GUO, E-mail: guodan26@mail.tsinghua.edu.cn 
also for lubrication in mechanical systems, which may lead to severe premature failures [8-10].

The effect of water on lubrication systems can be divided into two categories: long-term and instantaneous effects. Long-term effects include changes in the physical and chemical properties of lubricating oil and corrosion of the metal contact pairs [11-14]. Short-term effects are the instantaneous changes in the lubricating characteristics of the oil after water enters the lubricating oil, such as a decrease in viscosity and the weakening of the film-forming ability, which causes wearing of the metal contact pair. Presently, research on the effect of water on lubricating systems mainly focuses on changes in the long-term physical and chemical properties of the lubricating oil [15-17]. However, before the water changes the physical and chemical properties of the lubricating oil, it can change the lubrication characteristics of the contact area [18-21]. In addition to little research on the lubrication characteristics of emulsions, there are few direct observations and research on the influence of free water on the lubrication characteristics of the lubrication contact area. In this study, interference and infrared microscopy were used to directly observe the interaction between free water and the oil reservoir in the point contact area. The results can play a theoretical guiding role in exploring the early failure mechanism of the mechanical components caused by free water, and carrying out the protection work of water pollution.

This study mainly examines the state of oil starvation caused by micro oil supply. One of the significant reasons is that the oil starvation state is the most common working state of grease lubricated bearings because most of the grease is squeezed to both sides of the rolling track in the work, and the lubrication of the contact area is realized by the reflow of the oil separated from the grease on both sides of the contact area [22]. Moderate oil starvation reduces the heating of the bearing and lubricating grease and improves the bearing life. The bearing fails only when the oil starvation is severe. There are many similarities between oil and grease lubrication in the starvation state. Therefore, it is of great significance to evaluate the influencing factors of the oil starvation state under the condition of micro oil supply, and explore the influence of free water on the degree of starvation, not only for the mechanical parts working in the starved lubrication, but also for the design and use of grease lubricated bearings.

There are two lubrication conditions in the point contact structure of micro oil supply: fully flooded and starved. In this study, further research will be conducted on the influence of free water on oil lubrication characteristics in the contact area under these two lubrication conditions to further reveal the influence mechanism of free water on the film-forming characteristics with the measurement and analysis of the oil reservoir, rolling track in the contact area, and reflow characteristics.

\section{Experimental device and method}

A schematic diagram of the experimental device is shown in Fig. 1. In the experiment, a ball-on-disk test apparatus with an optical interference microscope was used to measure the influence of free water on the film thickness, inlet distance of the oil reservoir, track width, and reflow speed. When the amount of oil supply is fixed, the degree of starvation becomes severe with the increase in speed. In this study, PAO8 is used as a tested oil sample with a moderate viscosity of $50 \mathrm{~mm}^{2} / \mathrm{s}\left(40{ }^{\circ} \mathrm{C}\right)$ and good reflow characteristics, which can realize a smooth transition from the condition of fully flooded to starvation under micro oil supply; it is selected as the lubricant for the present study. For our experimental device, a $10 \mu \mathrm{L}$ oil supply is the most ideal, and can realize the transition from fully

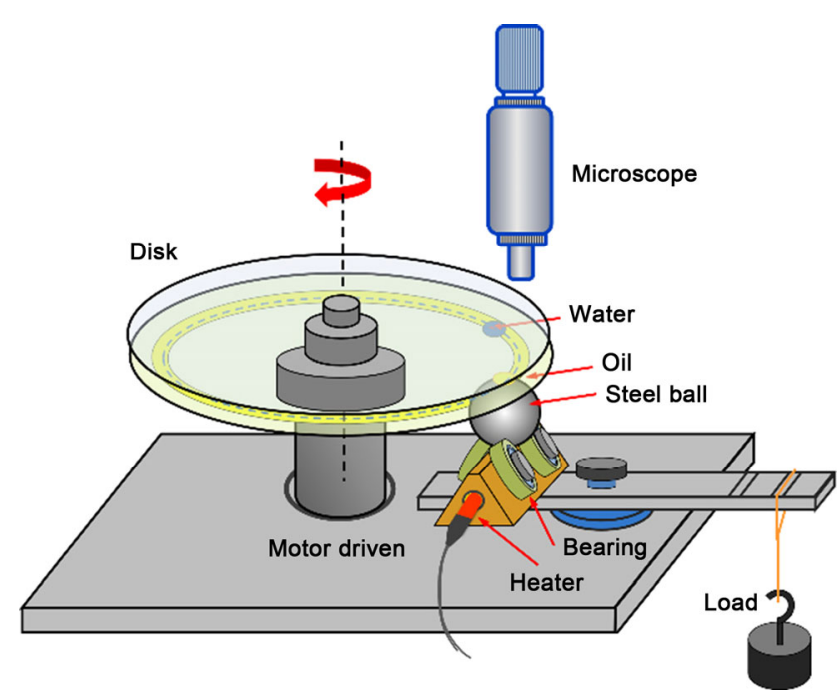

Fig. 1 Schematic diagram of the experimental setup. 
flooded to starvation in the most stable speed range of $0-1 \mathrm{~m} / \mathrm{s}$. Speeds of 100,350 , and $700 \mathrm{~mm} / \mathrm{s}$ are chosen as the representative speeds of the fully flooded, moderately starved, and severely starved lubrication conditions, respectively.

In the experiment, the ball was supported by three bearings mounted on top of a pedestal. The pedestal was mounted on one side of a lever, and a weight was placed on the other side of the lever to control the load applied. After loading between the ball and disk, the rotation of the glass disk drives the steel ball to rotate in a nearly pure rolling manner, and the steel ball also drives the three support bearings to rotate smoothly.

For the study of starvation, the main challenge is to precisely control the oil supply and evenly coat the little amount of lubricating oil on the track between the ball and disk. As shown in Fig. 1, when the load was applied, a wedge-like gap formed between the ball and plate. After starting the motor, approximately $10 \mu \mathrm{L}$ lubricating oil is evenly applied on the track, and a $2 \mu \mathrm{L}$ water droplet is added to the lower surface of the glass pan in the same track as the oil reservoir after the oil reservoir and film thickness in the contact area are stable. When the motor drives the glass disk to rotate, the water droplet is carried by the glass disk and moves towards the oil reservoir at the same speed. The effects of the water droplets on the lubrication conditions and film thickness at different speeds were observed. The white light microscope and high-speed camera mounted on the system recorded the entire process of water droplets entering the oil reservoir through a transparent glass disk; the image acquisition frequency was $100 \mathrm{fps}$. In the experiment, the load was $10 \mathrm{~N}$, the corresponding Hertz contact pressure was $550 \mathrm{MPa}$, and the Hertz contact radius between the ball and disc was $100 \mu \mathrm{m}$. The experiment was performed at $25^{\circ} \mathrm{C}$, and the relative humidity was $30 \%$. The film thickness obtained in the experiment has good repeatability, and the subsequent film thickness data are obtained from the average value of two measurements.

Because the working temperature of the bearings can reach $180{ }^{\circ} \mathrm{C}$, the invading process of free water into the oil reservoir at $2 \mathrm{~mm} / \mathrm{s}$ was also observed at $180{ }^{\circ} \mathrm{C}$ using an infrared microscope, to study the practical effect of free water in high-temperature contact pairs. A heater was inserted into the pedestal to control the temperature. Detailed information on the procedure for high-temperature heating and monitoring can be found in Ref. [23].

\section{Experimental results}

\subsection{Effect of free water droplets on the oil reservoir at different operating speeds}

First, the influence of water droplets on the oil reservoir in the contact area under different lubricating conditions is discussed. This section is significant for understanding the flow and film formation mechanism of the oil-water separated phase in and around the contact area.

As shown in Fig. 2, there is a certain depression on the meniscus at the entrance of the oil reservoir when the speed reaches $100 \mathrm{~mm} / \mathrm{s}$; however, the inlet distance is still $260 \mu \mathrm{m}$ from the boundary of the contact area, so the contact area is still in a fully flooded state at this time. Subsequently, a $2 \mu \mathrm{L}$ water droplet is added to the lubricating oil track on the lower surface of the glass disk. The water droplet rushes to the oil reservoir and gets stuck in its depression. However, the wettability of the water droplet to the solid wall is considerably less than that of the lubricating oil; therefore, the water droplet cannot directly enter the oil reservoir under the effects of the flow field of the oil reservoir and the centrifugal force. Finally, the water droplet stays on the rolling track and constantly washes the side of the

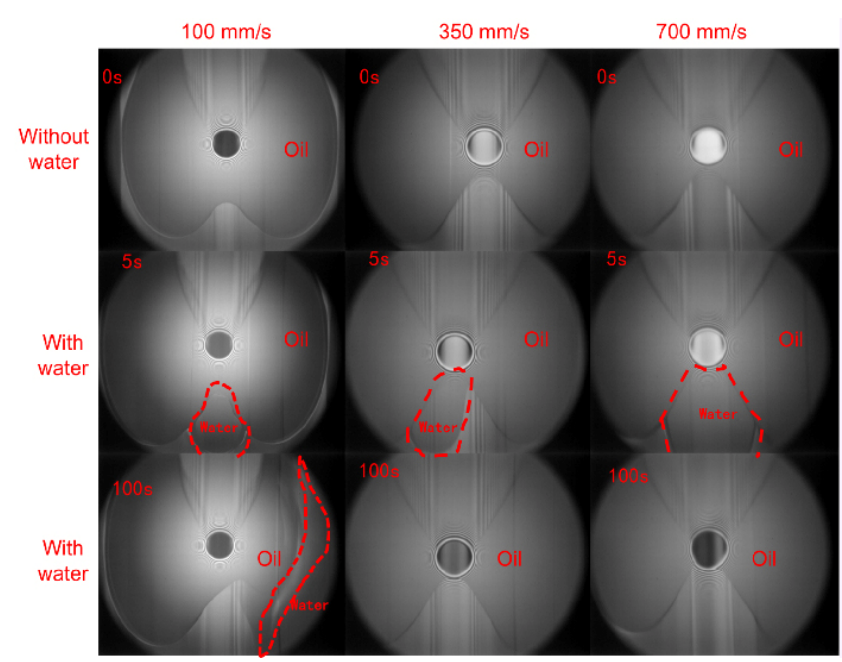

Fig. 2 Influence of a $2 \mu \mathrm{L}$ water droplet on the oil reservoir under different lubricating conditions. 
oil reservoir, which slightly reduces the volume of the oil reservoir. Accordingly, the inlet distance reduces to $215 \mu \mathrm{m}$.

When the speed is increased to $350 \mathrm{~mm} / \mathrm{s}$, the inlet distance of the oil reservoir to the contact is reduced to only $50 \mu \mathrm{m}$. Similarly, a $2 \mu \mathrm{L}$ water droplet is added to the rolling track of the glass disk. At this time, the water droplet directly breaks through the oil meniscus and passes through the contact area. With successive shearing, the entire droplet gradually decreases in size, and finally disappears from the field of view. The inlet of the meniscus, which is close to the edge of the contact area, completely goes into the contact area after being washed by the water droplet.

When the speed increases to $700 \mathrm{~mm} / \mathrm{s}$, the inlet meniscus of the oil reservoir moves to the contact, indicating that the contact is likely under severe starved conditions. A water droplet of $2 \mu \mathrm{L}$ is added again to the rolling track of the glass disk. Because there is almost no lubricant connecting the two parts of the oil reservoir, the water droplet easily enters the contact area under the shear force and is quickly "consumed" by successive shearing. From the captured photos, the gray value of the contact area drops significantly, indicating that the contact area is severely starved.

\subsection{Effect of water droplet on the film thickness under different lubricating conditions}

To further analyze the evolution of the lubricating conditions under the influence of a water droplet the central film thickness of the contact is calculated in Fig. 3.

The lubricating conditions with and without water droplets can be evaluated according to the Hamrock and Dowon equation [24]. A dimensionless parameter $m^{*}$ is used to define the critical state between the fully flooded and starved conditions.

$$
m^{*}=1+3.06 \cdot\left(\left(R_{1} / a\right)^{2} \cdot H\right)^{0.58}
$$

where $R_{1}$ and $a$ are the radii of the ball and contact, respectively. $H=h_{\mathrm{c}} / R_{1}$, which is the ratio of the central film thickness over the radius of the ball. If $D+a \geq a \cdot m^{*}$, the contact is fully flooded; if $D+a<$ $a \cdot m^{*}$, the contact is starved. $D$ represents the inlet distance.

At $100 \mathrm{~mm} / \mathrm{s}$, the calculated $D+a=360 \mu \mathrm{m}$, which is considerably larger than $a \cdot m^{*}=101 \mu \mathrm{m}$; therefore, the contact is under the fully flooded condition. The film thickness difference between the two cases (with and without free water) is less than $3 \mathrm{~nm}$. The presence of the free water droplet does not change the lubricating condition in this case. Therefore, it can be deduced that at a relatively low rolling speed, the lubricating oil can form a complete and stable oil reservoir around the contact area. A water droplet can neither remain stable in the inlet area, nor break the oil meniscus and go through the contact.

At $350 \mathrm{~mm} / \mathrm{s}$, the calculated $D+a=150 \mu \mathrm{m}$ and $a \cdot m^{*}=115 \mu \mathrm{m}$. Therefore, the contact is still under the fully flooded condition; however, it is under a progressive lubricant starvation condition. In this case, it is observed that the water droplet has an impact as it passes through the contact. Subsequently, the inlet meniscus of the oil reservoir moves into the contact, and the central film thickness drops rapidly from $120 \mathrm{~nm}$ to approximately $80 \mathrm{~nm}$, which indicates that the contact enters the starved condition.

At $700 \mathrm{~mm} / \mathrm{s}$, the calculated $D+a=100 \mu \mathrm{m}$ and
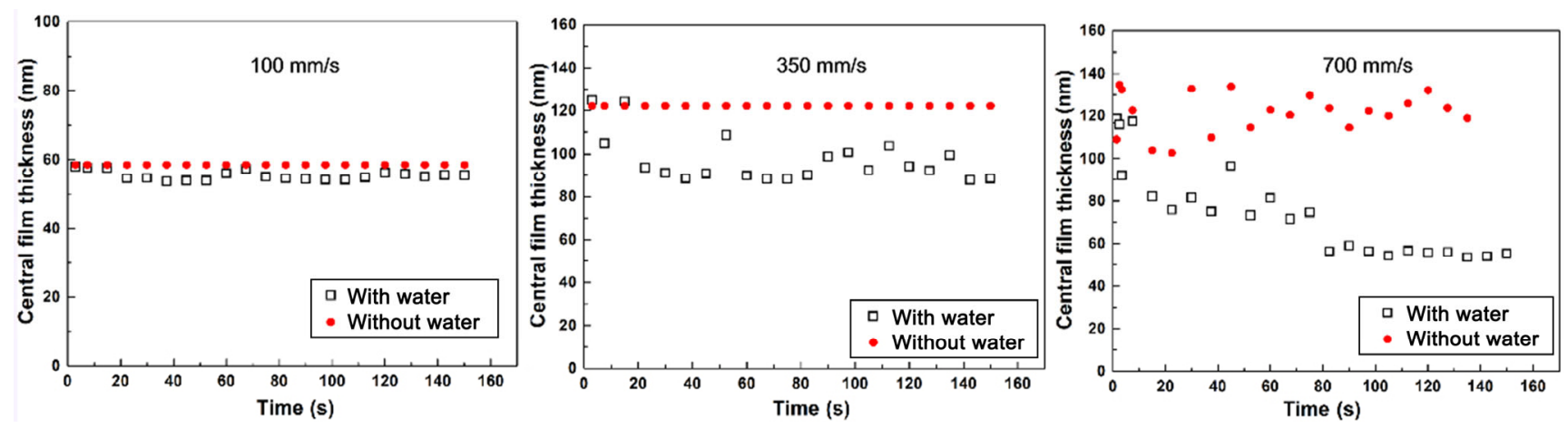

Fig. 3 Influence of a $2 \mu \mathrm{L}$ water droplet on the film thickness under different lubricating conditions. 
$a \cdot m^{*}=115 \mu \mathrm{m}$. The oil meniscus of the oil reservoir moves into the contact even in the case without water droplets, which indicates that the contact is starved. When the water droplet is added, the film thickness drops sharply from 120 to $50 \mathrm{~nm}$. The contact area is in a state of severe starvation, and the destructive effect of the free water droplet on the film-forming ability of the oil is more significant.

\section{Discussion}

The mechanism of film formation and displacing effect of a water-in-oil emulsion has been studied extensively $[6,7]$. For a water-in-oil emulsion lubricating system under fully flooded conditions, the basic film thickness is maintained by the oil, while the water particles may increase the viscosity of the lubricant, which contributes to the increase in film thickness. However, in the present study, limited oil is added to the disc, and only one large water droplet separated from the oil phase is trying to enter the contact. The viscosity of the lubricating system does not change, and the amount and dimensions of the water droplets and oil are comparable. Under this condition, the mechanism can be more complex at the air-oil-water interface.

In this section, the mechanism of the effect of free water on the film-forming ability is further discussed. From the above experiments, three stages of the water droplet entering the contact can be determined as follows: (1) First, the free water droplet rushes to the oil reservoir and is stuck at its entrance, which significantly affects the reflow caused by the capillary force at the inlet of the contact area. However, this reflow part is the main part of the lubrication supplement under the starved condition; (2) when the inlet distance is relatively short, the free water droplet breaks through the oil film in the entrance area, and is gradually sheared. Under the effect of contact pressure, the water penetrates the oil film contact, thus replacing the oil layer in the track; (3) with continuous shearing, an increasing number of water molecules remain inside the track until an equilibrium is reached. Additionally, the existence of a water component inside the track significantly reduces the reflow ability of the oil. Although the proportion of the reflow in the track outside the contact area is small, the change in surface tension of the track also affects the reflow ability around the contact area.

Detailed analysis and more evidence on the proposed mechanism are given as follows.

\subsection{Effect of free water on the reflow at the entrance of the oil reservoir}

In the experiment setup, the stable oil reservoir near the contact area is the result of the dynamic balance between the rolling loss and reflow supply. Therefore, regardless of the reflow in the oil reservoir or the reflow in the rolling track outside the contact area, it cannot be directly observed under dynamic experimental conditions $[25,26]$. However, we can still observe the reflow near the contact area and in the rolling track outside the contact area by stopping the motion of the friction pair. We can also determine the effect of the free water droplet on the lubrication replenishment process, which is affected by capillary and van der Waals forces from the observed reflow phenomenon [27-30].

Figure 4 shows the capillary reflow process of the oil at the entrance of the contact area after stopping the motor. It can be observed that the capillary effect of the lubricating oil is obvious because the space near the contact area is small. After the motor stops, the two parts of the oil reservoir quickly close from both sides to the middle. Within $1 \mathrm{~s}$, the rolling track in front and behind the contact area is completely

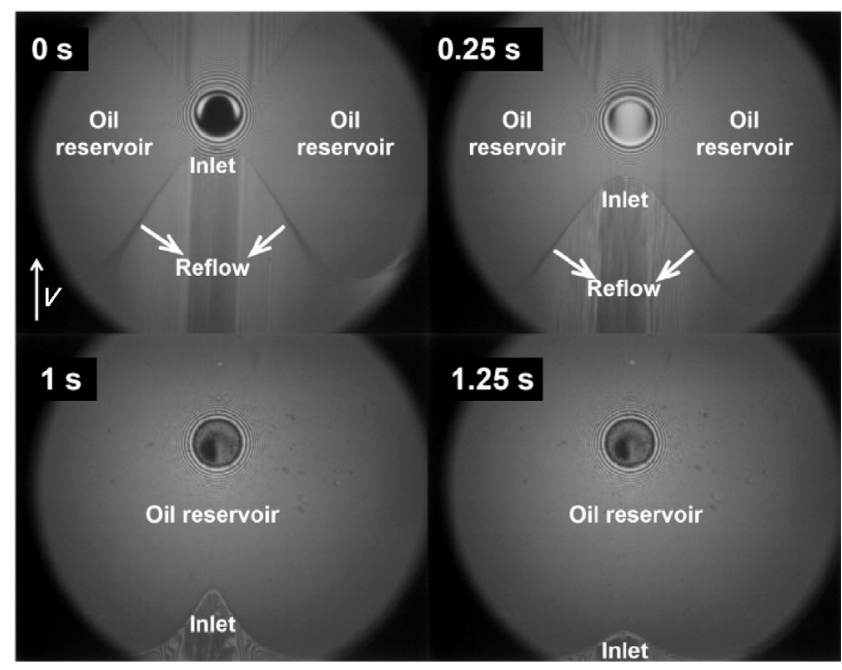

Fig. 4 Process of lubricating oil reflow back to the inlet of the contact area. 
filled, and a round oil reservoir is recovered. It can be concluded that capillary reflow plays a significant role in the dynamic starved lubrication process from the time required for capillary reflow after the motor stops.

Figure 5 shows a comparison of the rolling tracks affected by water droplets at different speeds. In the previous study, the inlet distance at the entrance was $210 \mu \mathrm{m}$ at a speed of $100 \mathrm{~mm} / \mathrm{s}$, and the contact area was under the fully flooded condition. The water droplet could not break through the oil meniscus in the inlet area, thus it could not enter the contact area. Therefore, after a sudden halt of the ball and disc, oil reflowed rapidly back to the track, indicating that the nature of the oil and rolling track changed slightly. When the rolling speed exceeded $350 \mathrm{~mm} / \mathrm{s}$, the inlet distance of the oil reservoir decreased to less than $50 \mu \mathrm{m}$, and the water droplet passed through the oil meniscus and entered the contact. Afterwards, the large water droplet was sheared into smaller droplets because of the high contact pressure; the water and oil mixture adhered onto the entire rolling track. The presence of water content on the track surface significantly prevented oil from reflowing back. The consequence was that the boundary between the rolling track and oil along both sides was still clearly visible even after a considerable period of time, i.e. several minutes. At a higher speed of $700 \mathrm{~mm} / \mathrm{s}$, the boundary of the track was clear, and another intermediate state of emulsion occurred in the middle of the track and oil phase. It can be speculated from the captured image that the emulsification is more like the oil in water emulsion because the boundary between the oil and emulsion phases is distinct.

\subsection{Decisive factor of a water droplet to enter a lubricating contact}

For the case of the $10 \mu \mathrm{L}$ oil supply, it is found that the critical condition for the free water droplet to

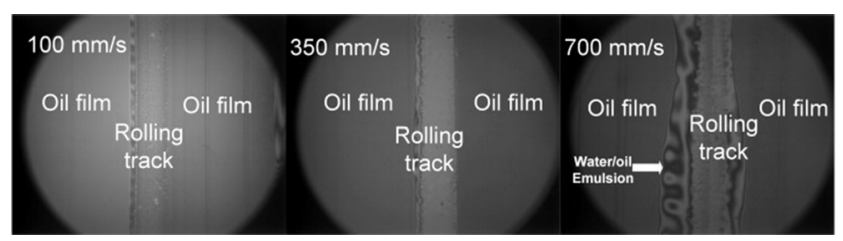

Fig. 5 Comparison of the oil film inside and outside of the rolling track at different speeds. penetrate into the contact area is a speed of $350 \mathrm{~mm} / \mathrm{s}$ and inlet distance of $50 \mu \mathrm{m}$. However, it is still unclear which of the two critical conditions is dominant, leading to the water droplet entering the contact area. If the speed is the decisive condition, then by increasing the oil supply and inlet distance, the free water droplet should also pass through the contact area at a speed of $350 \mathrm{~mm} / \mathrm{s}$. However, if the inlet distance is the decisive condition, the water droplet should pass through the contact area at a lower speed by reducing the oil supply. To answer this question, further experiments are carried out as follows.

The rolling speed was maintained at a constant of $350 \mathrm{~mm} / \mathrm{s}$, and oil supply was increased from 10 to $15 \mu \mathrm{L}$. The results are shown in Fig. 6. With $15 \mu \mathrm{L}$ of oil supply, the stabilized inlet distance of the oil reservoir increases to $150 \mu \mathrm{m}$. At this time, the added water droplet only stays at the entrance for a short period of time, but cannot break through the oil meniscus. After a while, the water droplet slips out of the inlet area and remains outside the oil reservoir. However, with a $10 \mu \mathrm{L}$ oil supply, the inlet distance is only $50 \mu \mathrm{m}$, and the free water drop can break through the oil film in the inlet and enter the contact area.

The inlet distance was then maintained at $50 \mu \mathrm{m}$. A $50 \mu \mathrm{m}$ inlet distance was achieved at a speed of $100 \mathrm{~mm} / \mathrm{s}$ by reducing the total oil supply to $5 \mu \mathrm{L}$. The experimental results are shown in Fig. 7. As long as the inlet distance is maintained at $50 \mu \mathrm{m}$, even when the rolling speed decreases to $100 \mathrm{~mm} / \mathrm{s}$, the free water

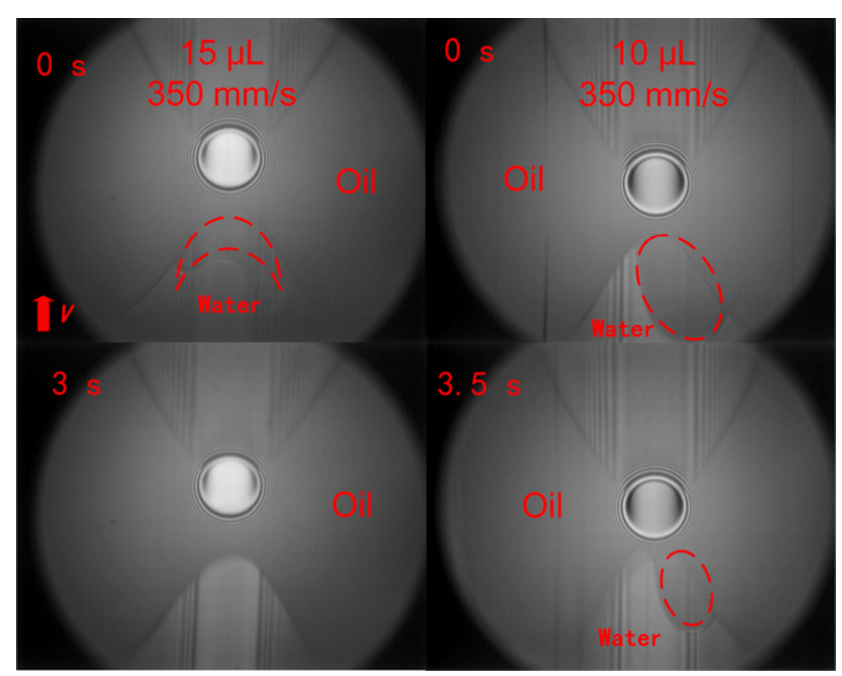

Fig. 6 Impact of water on the inlet of the reservoir with different amounts of oil supply at a constant speed of $350 \mathrm{~mm} / \mathrm{s}$. 


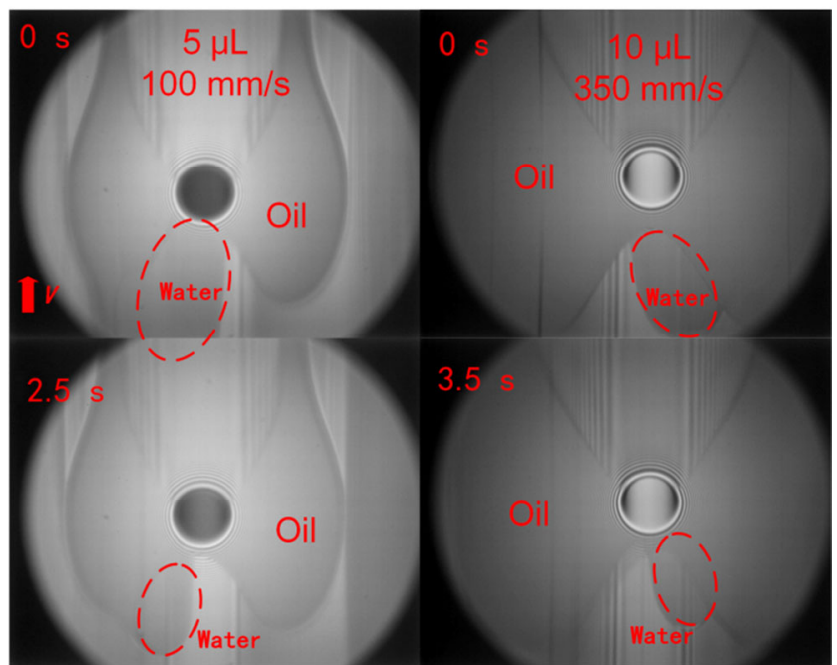

Fig. 7 Water droplet impact on the inlet of the oil reservoir at different speeds with constant inlet distance of $50 \mu \mathrm{m}$.

droplet can still pass through the contact area in $3 \mathrm{~s}$, which decreases the film thickness.

Based on the above analysis, the inlet distance is the decisive factor for the free water droplet to enter the contact area. It should be noted that the critical inlet distance may change according to the size of the water droplet, oil viscosity, and size of the contact area, which is of interest and will be studied in future work.

In this study, the critical condition of a water droplet of $2 \mu \mathrm{L}$ to enter the contact area at different oil supply values is summarized in Table 1. The inlet distance is the decisive factor determining whether the water droplets can pass through the contact area. The ratio of the critical inlet distance $S_{\text {Inlet }}$ and the radius of the contact area $R_{\text {Hertz }}$ is as follows:

$$
I=S_{\text {Inlet }} / R_{\text {Hertz }}=0.5
$$

When the ratio is less than this critical value, the free water droplet can break through the lubricating film connecting the two parts of the oil reservoir and entering the contact area. The inlet pressure drop

Table 1 Critical condition of water droplet to enter the contact area under different oil supply values.

\begin{tabular}{cccc}
\hline $\begin{array}{c}\text { Oil supply } \\
(\mu \mathrm{L})\end{array}$ & $\begin{array}{c}\text { Critical speed } \\
\left(\mathrm{mm} \cdot \mathrm{s}^{-1}\right)\end{array}$ & $\begin{array}{c}\text { Critical inlet } \\
\text { distance }(\mu \mathrm{m})\end{array}$ & $\begin{array}{c}\text { Contact } \\
\text { radius }(\mu \mathrm{m})\end{array}$ \\
\hline 15 & 500 & 50 & 100 \\
10 & 350 & 50 & 100 \\
5 & 100 & 50 & 100 \\
\hline
\end{tabular}

caused by the low viscosity of water and the sheared free water can also continuously wash the oil on the rolling track, causing the film thickness to drop sharply.

\subsection{Emulsification of oil by free water under starved condition}

Owing to the shear effect of the contact zone, the free water droplet is divided into small droplets, which combine with the lubricating oil to form a water/oil emulsion. This emulsion also affects the lubrication characteristics of the contact area.

Figure 8 shows the comparison results of the oil reservoir with and without the impact of the water droplet at different speeds. At $100 \mathrm{~mm} / \mathrm{s}$, the water droplet does not affect the oil reservoir; at $350 \mathrm{~mm} / \mathrm{s}$, several large water droplets are observed in the oil reservoir around the contact zone, which proves that the water droplet can be sheared apart after passing through the contact; at $700 \mathrm{~mm} / \mathrm{s}$, the free water droplet is completely sheared into a large number of small water droplets (approximately less than $10 \mu \mathrm{m}$ in diameter), and dispersed evenly throughout the oil reservoir. This process is similar to the emulsification process. Furthermore, the small droplets can stably remain in the oil reservoir without gathering during our observation.

According to Ref. [6], for a water/oil emulsion lubricating system under the fully flooded condition, the water particles may increase the viscosity of the lubricant, which contributes to the increase in film thickness. However, the present results show that the central film thickness might decrease for such lubricating systems if the contact is not fully rinsed in the oil bath, or the contact is under starved conditions. It is worth noting that in Ref. [6], the entire emulsion system was pre-made before the experiment was conducted; however, in the present study, the final emulsion system was gradually formed during rolling and shearing. These are two different flow dynamics

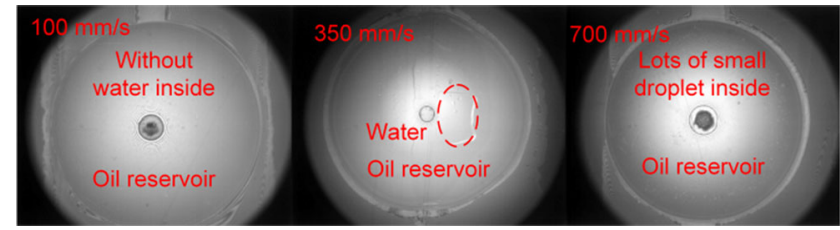

Fig. 8 Influence of water on the oil reservoir under different speeds. 
and lubrication mechanisms, which cannot be compared directly.

\subsection{Water droplet invading superheated reservoir}

The process of a water droplet invading the superheated oil reservoir is shown in Fig. 9. The water droplet sprays and evaporates violently as soon as it touches the high-temperature ball. Although many water droplets splash on the lower surface of the disk around the oil reservoir, most of them still enter the oil reservoir.

The diffusion of the water droplets in the superheated oil reservoir is shown in Fig. 10. The water close to the atmosphere gradually decreases and contracts to the oil reservoir because of the outward diffusion of molecules. After $3 \mathrm{~min}$, the two water droplets decrease gradually and completely contract into the oil reservoir. The oil has an enormous resistance to the diffusion of the water droplets inside it. Although the temperature of the contact zone is considerably higher than the boiling point of the water, these small water droplets can exist stably in the superheated oil reservoir for a long time, which is bound to cause long-term damage to the lubrication formation of the mechanical system.

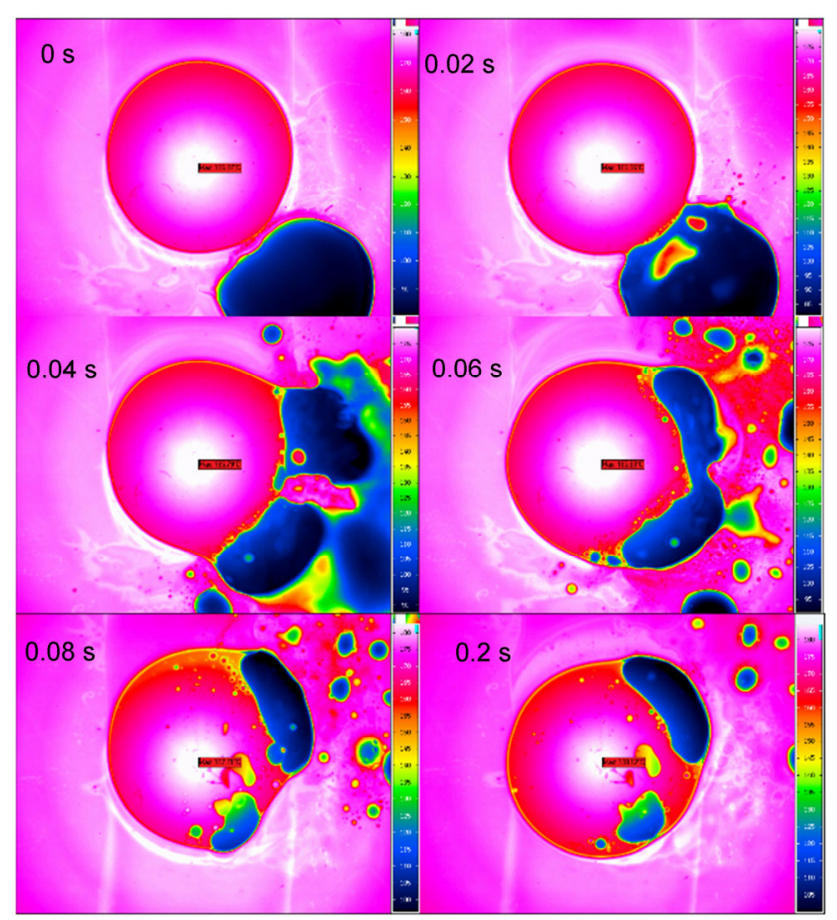

Fig. 9 Process of water drop invading superheated oil reservoir.

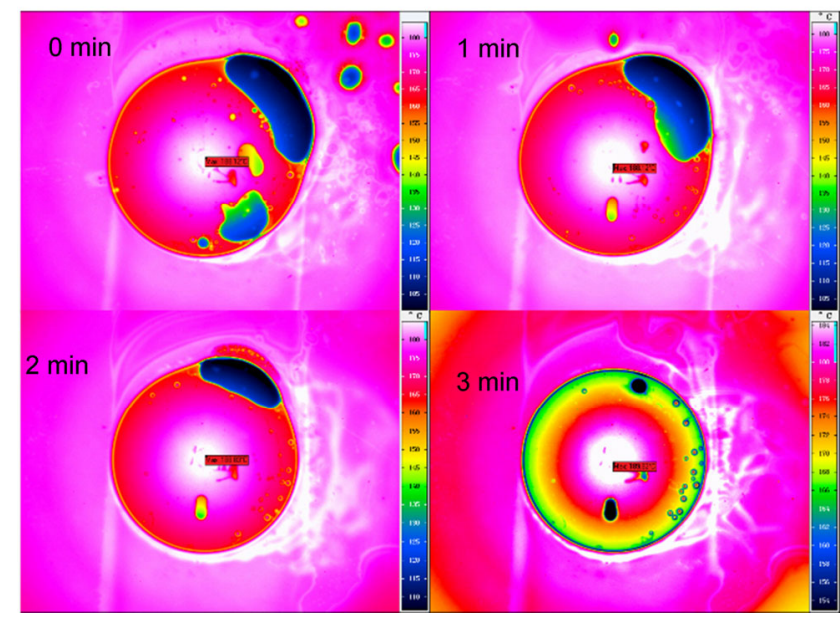

Fig. 10 Diffusion of water droplets in the superheated oil reservoir.

\section{Conclusions}

According to the results and analysis of the film thickness and oil reservoir characteristics, the influence of free water on film-forming characteristics under the condition of micro oil supply can be summarized as follows:

1) When a free water droplet rushes (entering) to the contact area under progressive or catastrophic lubricant starved conditions, it is stuck at the entrance of the oil meniscus, which significantly affects the reflow caused by capillary action at the entrance. This causes or aggravates contact starvation.

2) The ratio between the inlet distance and radius is the decisive factor in determining whether a water droplet can pass through the contact area. In the present study, when this ratio was less than $1 / 2$, the free water droplets broke through the oil film, and were gradually sheared into the contact. The sheared water droplets continuously washed the track, reducing the film thickness. When the lubricant starvation was progressive, only the oil film adhered on the track was involved in the film formation; therefore, the water drop erosion significantly increased the starvation in the contact area.

3) Free water droplets can be sheared into small water droplets, and the oil reservoir is emulsified into a water/oil emulsion system. It was observed that the central film thickness of the contact decreased under such conditions. 
4) After the water droplet invades the superheated oil reservoir, it is easy to form a partially fused water/ oil droplet. Significant inhibition of the oil film on the evaporation and diffusion of water molecules in the superheated system was observed. These small water droplets can exist stably in the oil reservoir for a long time, which is bound to harm the lubrication of the mechanical system.

In conclusion, the existence of free water in lubricating oil can be harmful even before the change in physical and chemical properties such as acidification and oxidation, particularly under EHD starvation lubrication conditions, which further aggravate the degree of contact starvation, thus sharply reducing the EHD film thickness and causing wear.

\section{Acknowledgements}

This work was financially supported by the National Natural Science Foundation of China (Grant Nos. 51805505, 52075514, and 51605460).

Open Access This article is licensed under a Creative Commons Attribution 4.0 International License, which permits use, sharing, adaptation, distribution and reproduction in any medium or format, as long as you give appropriate credit to the original author(s) and the source, provide a link to the Creative Commons licence, and indicate if changes were made.

The images or other third party material in this article are included in the article's Creative Commons licence, unless indicated otherwise in a credit line to the material. If material is not included in the article's Creative Commons licence and your intended use is not permitted by statutory regulation or exceeds the permitted use, you will need to obtain permission directly from the copyright holder.

To view a copy of this licence, visit http://creativecommons.org/licenses/by/4.0/.

\section{References}

[1] Needelman W M, Barris M A, LaVallee G L. Contamination control for wind turbine gearboxes. Power Eng 113: 112-112 (2009)

[2] Kotzalas M N, Doll G L. Tribological advancements for reliable wind turbine performance. Philos Trans $R$ Soc London, Ser A 368: 4829-4850 (2010)

[3] Terrell E J, Needelman W M, Kyle J P. Current and future tribological challenges in wind turbine power systems. In ASME/STLE 2009 International Joint Tribology Conference, Tennessee, USA, 2009: 495-497.

[4] Patchett B M, Yarmuch M A R. Hydrocarbon Contamination and Diffusible Hydrogen Levels in Shielded Metal Arc Weld Deposits. Welding Journal 89: 262-265 (2010)

[5] Fitch J C, Jaggernauth S. Moisture-The second most destructive lubricant contaminate, and its effects on bearing life. P/PM Technology 12: 1-4 (1994)

[6] Wan G T Y. Elastohydrodynamic film thickness of water based hydraulic fluids. Ph.D Thesis. London (UK): Imperial College University, 1983.

[7] Wan G T Y, Kenny P, Spikes H A. The elastohydrodynamic properties of water-based fire-resistant hydraulic fluids. Tribol Int 17: 309-315 (1984)

[8] Matter P. Seal oil contamination. Ind Lubr Tribol 35(3): 84-86 (1983)

[9] Johnson M, Spurlock M. Strategic oil analysis: Instrumentbased on-site lubricant analysis. Tribol Lubr Technol 66: 26-27 (2010)

[10] Johnson M, Spurlock M. Strategic oil analysis: Setting the test slate. Tribol Lubr Technol 5: 20-27 (2009)

[11] Zhu J. Online industrial lubrication oil health condition monitoring, diagnosis and prognostics. Ph.D Thesis. Chicago (USA): University of Illinois, 2013.

[12] Roddis A. Reducing moisture contamination in bearing lubrication. Sealing Technology 2: 6-9 (2006)

[13] Troyer D. Establishing moisture contamination targets for hydraulic systems. Machinery Lubrication 28-32 (2004)

[14] Moon M. How clean are your lubricants? Trends Food Sci Tech 18: 74-79 (2007)

[15] Johnson M. Lubricant management: Dynamic circulating systems. Tribol Lubri Tech 65: 32 (2009)

[16] Tiwari R, Khatana M. Lubrication oil replacement technology. IJRTET 7(3): 8-11 (2012)

[17] King R A, Mckehzie P. Microbial degradation of marine lubricating oils. Ind Lubr Tribol 29: 4-25 (1977)

[18] Cyriac F, Lugt P M, Bosman R, Venner C H. Impact of water on EHL film thickness of lubricating greases in rolling point contacts. Tribol Lett 61(3):1-8 (2016)

[19] Chen J, Yan F Y, Wang J Z. Corrosion wear properties of TC4 titanium alloy in artificial seawater. Tribology 32(1): $1-6(2012)$

[20] Chen $\mathrm{H}, \mathrm{Wu} \mathrm{F}$, Zhao W J, et al. Influence of epoxy value on the tribological performances of epoxy resin coatings in seawater environment. Tribology 34(6): 601-607 (2014) 
[21] Chen R L, Wang Y, Lei H. Flow characteristics of nanoconfined water under dynamic load by molecular dynamics simulation. Tribology 36(6): 673-678 (2016)

[22] Damiens B, Venner C H, Cann P M E, Lubrecht A A. Starved lubrication of elliptical EHD contacts. $J$ Tribol-T ASME 126: 105-111 (2004)

[23] Liu X, Huang L, Guo D, Xie G X. Infrared thermography investigation of an evaporating water/oil meniscus in confined geometry. Langmuir 33: 197-205 (2017)

[24] Ball bearing lubrication: The elastohydrodynamics of elliptical contacts. New York (USA): Wiley Interscience, 1981.

[25] Jacod B, Pubilier F, Cann P M E, Lubrecht A A. An analysis of track replenishment mechanisms in the starved regime. Tribology Series 36: 483-492 (1999)

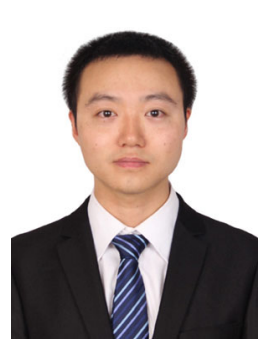

Xiang LIU. He received his Ph.D. degree in 2015 from State Key Lab of Tribology, Tsinghua University, Beijing, China. He studied the mechanism of oil lubrication during

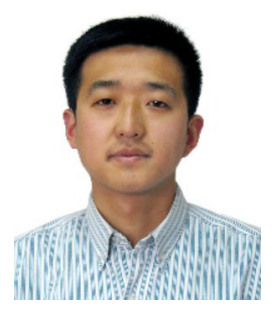

Jintao WANG. He received his bachelor, master, and doctor degrees from Xi'an Jiaotong University in 1998, 2001, and 2006, respectively.

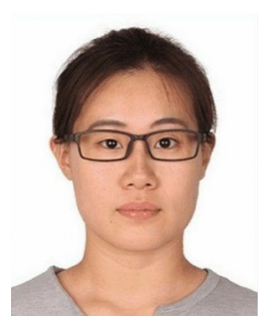

Lu HUANG. She received her Ph.D. degree in 2016 from State Key Lab of Tribology, Tsinghua University, Beijing, China. She studied the mechanism of grease and oil lubri-

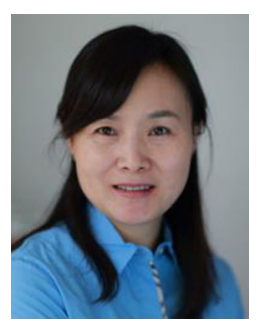

Dan GUO. She received the M.S. degree in engineering mechanics in 1995 from Xi'an Jiaotong University, Xi'an, China and Ph.D. degree in engineering mechanics in 1999 from Tsinghua University, Beijing, China. She joined the State Key Laboratory
[26] Hurley S, Cann P M, Spikes H A. Lubrication and reflow properties of thermally aged greases. Tribol Trans 43: 221-228 (2000)

[27] Cann P M, Lubrecht A A. The effect of transient loading on contact replenishment with lubricating greases. Tribology Series 43: 745-750 (2003)

[28] Cann P M E, Damiens B, Lubrecht A A. The transition between fully flooded and starved regimes in EHL. Tribol Int 37: 859-864 (2004)

[29] Gershuni L, Larson M G, Lugt P M. Lubricant replenishment in rolling bearing contacts. Tribol Trans 51: 643-651 (2008)

[30] Nagata Y, Kalogiannis K, Glovnea R. Track replenishment by lateral vibrations in grease-lubricated EHD contacts. Tribol Trans 55: 91-98 (2012)

Ph.D. period. Now he is an associate research fellow in National Institute of Metrology, Beijing, China. His research area mainly focuses on the volume and density metrology method.

He is working for Volume and Density Laboratory of National Institute of Metrology as a research fellow, and the main research field is volume and density metrology method.

cation during Ph.D. period. Now she is an associate research fellow in National Institute of Metrology, Beijing, China. Her research area mainly focuses on the evaluation of lubricants and matierials, and nanoparticle characterization.

of Tribology at Tsinghua University from 1999. Her current position is a professor and the deputy director of the laboratory. Her research areas cover the properties of friction at the micro/nano-scale, mechanism of interaction among nanoparticles and surface in ultra-smooth surface planarization, and the formation and lubricayion failure in harsh conditions. 